\title{
RT
}

\section{Choice cuts for patients with AIDS?}

\section{Contracts could be used to improve services for patients with AIDS - but the outlook is bleak}

Genitourinary medicine clinics have provided the cornerstone of public health strategy to control epidemics of sexually transmitted diseases since the Venereal Disease Regulations of 1916. Although genitourinary medicine has changed dramatically since the 1980s under the impetus of the HIV epidemic, this strategy still depends on providing a free, open access specialist service that guarantees confidentiality. The changes proposed in Working for Patients could threaten the ability of genitourinary medicine clinics both to care for patients with AIDS and to fulfil their public health functions.

At first glance genitourinary medicine clinics do not seem to be threatened by the changes. Contracts for Health Services: Operating Contracts confirms the aim of guaranteeing choice for patients, "particularly where services are provided on a walk in basis," and expects district authorities to fund genitourinary medicine clinics in the same way as accident and emergency departments, through "block" payments in advance for a defined range of services available to all, regardless of residency. ${ }^{1}$ Moreover, the Department of Health has recently tacitly accepted genitourinary medicine as a "core" or "designated" service by instructing regions and districts that they must implement the recommendations of the Monk Inquiry, ${ }^{2}$ one of which was that each district should offer a service for genitourinary medicine and HIV infection. ${ }^{3}$ What is not clear, however, is whether the extra costs of providing a service open to all comers will be funded over and above the district's capitation based budget. ${ }^{+}$

Since the 1980s there has been greater emphasis on the viral infections herpes, warts, and HIV. Caring for patients with these infections is much more complex and the resource implications greater. In particular, each new contact with a patient with HIV infection is the start of a relationship with a key professional who will continue to provide support for the rest of the patient's life. All this is a far cry from the "emergencies only" culture of the accident and emergency department. Can the same simplistic funding mechanism really apply to both?

Although the main contract with genitourinary clinics is likely to be a "block" contract, as with accident and emergency services, the position will change if patients need admission or referral to other outpatient departments. Then cost recovery will depend on the standard contracting process. This will be problematic. For patients with AIDS in particular contracts are unlikely to be lucrative and so will not be a managerial priority when beds are allocated. Patients will continue to refer themselves to convenient or well reputed clinics whatever the contractual relationship between the clinic and their particular district of residence. If no contract

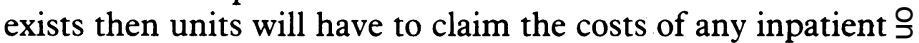
care on a cost per case basis from the district's contingency $\vec{G}$ reserve. Such a system would be costly in administrative time, $\infty$ retrospective, and unworkable on a large scale. When there $\frac{\mathbb{D}}{\mathbb{D}}$ are contracts, purchasing authorities may not be prepared to $\frac{\mathbb{D}}{3}$ pay for the level of service presently offered in specialist units $\underset{\mathbb{D}}{\mathbb{D}}$

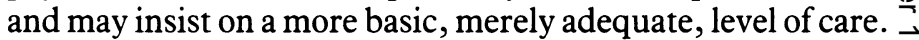

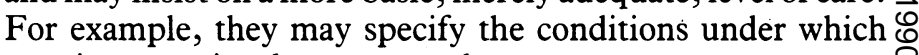
certain expensive drugs are used.

The most feasible solution would be for provider units to have contracts directly with regional health authorities. The money could either continue to come to them directly from the Department of Health - this would be preferable because $\frac{}{\square}$ of the uneven geographical spread of cases of $\operatorname{AIDS}^{5}$ (although $\stackrel{\varrho}{\rightleftharpoons}$ even now there are immense variations in cost per case between regions (p 509)) - or be "top sliced" from regional allocations.

The complexity of contracts will be at its worst in the main centres dealing with HIV infection, most of them in teaching districts. Up to eight different types of contract might have to be raised if the current range of service is to be maintained, 3 . some of them with several different districts or regions. $\delta$ There are also problems in defining district of residence and $₹$ preserving anonymity. ${ }^{6}$ Yet if confidentiality is compromised $ᄋ$ in the interests of accountability patients will not be encour- N aged to present early. Many patients with AIDS move house $\rightarrow$ while receiving treatment, often to be nearer to the health services that provide them with support. Although their original district continues to carry financial responsibility for $\mathrm{W}_{\mathrm{W}}$ three months after a move, districts providing a high level of 0 service for such patients will have to accept the financialo consequences of attracting heavy users on to their patch. ${ }^{6} \mathbb{\Phi}$ Even more difficult issues are raised by the homeless, a ? growing group in clinics serving inner cities.

Nor is it clear what will happen to the "outreach" agencies $\stackrel{0}{\mathbb{D}}$ such as the Terrence Higgins Trust and Frontliners, whose $\frac{\rho}{\mathbb{P}}$ work is so important to patients with HIV infection. They will $\frac{\varrho}{\sigma}$ have to seek funds from district health authorities on a contractual basis in competition with statutory services. $\frac{0}{0}$ Similarly, statutory schemes such as needle exchanges and the Central London Action on Street Health team will be vulner- $\frac{\bar{u}}{\bar{P}}$ able to cost cutting. Like the voluntary bodies, they encourage the use of services as well as providing education and advice on prevention. Will districts with tight budgets be 
keen to continue sponsoring activities that result in greater use of their services?

The tailored package of care and support for individual patients that has been created in some areas in response to the HIV epidemic would serve as a model for the care of patients with other chronic conditions. This system is threatened in three ways: general practitioners will be reluctant to take on patients with HIV infection or AIDS because they need costly drugs and create a heavy workload; acute and community services in the same district will be managerially separate and their relationships complicated by cross charging; and community care teams will have to agree contracts with districts of residence before crossing boundaries to facilitate continuity of expert care for patients.

In outline these are some of the problems for one specialty. Nevertheless, there may also be opportunities to modify the original plans to make them more workable. Indeed, the contracting process could be used to improve the service for patients with AIDS. If regional health authorities contract for AIDS services directly they could programme development to "roll out" new knowledge and skills from the few specialist centres to non-teaching districts. Centres with academic and research contracts could continue their research and development with extra regional funding to allow for their more complex case mix. "Satellite" districts would then hold contracts with the specialist centre for a form of shared care. These would be based on protocols, negotiated between clinicians, specifying the level of work to be handled in each district if the patient was willing. An additional strategy would be for consultants from the specialist centres to take on sessions with other district clinics, and vice versa. Just as important, the contracts could also specify the training commitments necessary for systematically disseminating knowledge and skills. Some such mechanism is vital as the numbers of patients will soon become too great for just a few centres to manage.

It is a great worry that the NHS review may threaten the quality and quantity of provision of services for genitourinary medicine and HIV infection. Urgent further thought must be given to supporting their open access systems for reasons of personal and public health. Not only must they survive, but they must be encouraged to develop and expand to meet the rapidly growing demands for their complex services. Otherwise many clinics and departments will contract that increasingly prevalent strain of AIDS - the acute income deficiency syndrome - which in the free market world has a negligible survival rate.

CHRIS BENTLEY Senior Registrar MICHAEL W ADLER Professor of Genitourinary Medicine

Academic Department of Genitourinary Medicine,

University College and

Middlesex School of Medicine,

London WIN 8AA

Department of Health. Working for patients. Contracts for health services: operating contracts. London: HMSO, 1990.

2 Department of Health. HIV and AIDS: resource allocations 1990/91. London: DoH, 1990 $(\mathrm{EL}(90) \mathrm{P} / 30$.

3 Working group to examine workloads in genitourinary medicine clinics. Report. London: DoH 1988. (Chairman A Monk.)

4 Department of Health. District allocations. London: DoH, 1990. (EL(90)MB/22.

5 Department of Health. Working for patients: WP2 funding and contracts for hospital services. London HMSO, 1989.

6 Department of Health. Contracts for health services: operational principles. London: DoH, 1989.

\section{Children with diabetes}

\section{Every health district should have a specialist clinic for their care}

The ravages of diabetic complications are not seen in a children's diabetes clinic. Nevertheless, they haunt the paediatrician who believes that sustained control of the blood glucose concentration will protect today's child from dying 20 years hence with renal failure, partially blind, and with ulcerated neuropathic feet. ${ }^{1}$

Diabetes in a child pervades all aspects of family life. The essential components of care are straightforward enough balancing insulin and diet with the day's activities - but the orchestration of the life of the diabetic child with the aim of achieving near normal glycaemia is a demanding business for all concerned: the child, the family, and the clinic team. Might the burden of care be lightened by more specialised attention and might normoglycaemia then be achieved, offering a better deal for adult life? A recent study by a working party of the British Paediatric Association goes some way towards answering these questions. ${ }^{2}$

Swift et al sent a questionnaire to all 820 consultant paediatricians who were members of the British Paediatric Association in March 1988 to study the services for children with diabetes in Britain. Replies were received from $93 \%$ of the consultants, giving information from 205 of the 220 health districts or boards. The answers showed that though the services for children with diabetes were well organised in some districts, there were substantial deficiencies in the network of care. Just over one quarter of districts did not have a designated specialist clinic for children with diabetes; $19 \%$ of consultants worked in districts with no support from diabetic nurse specialists or health visitors (and most only had such nursing support to a marginal degree); $9 \%$ of clinics did not provide regular measurements of glycated haemoglobin or fructosamine concentration; $63 \%$ did not provide the support of a trained paediatric dietitian; and $30 \%$ did not have a dietitian in attendance. In $13 \%$ of clinics children were seen regularly by senior house officers, and $45 \%$ of clinics did not offer out of hours support by telephone from specialist nurses or consultants for the children's families.

The scene is patchy and falls short of standards recently published by the British Diabetic Association for the specialist supervision of children with diabetes. ${ }^{3}$ The working party's report identifies the paediatricians who can be regarded as specialists in diabetes: they run large clinics specifically for diabetic children and their families. It is in association with these 100 specialist clinics that we find, predictably perhaps, the additional services of specialist nurses or health visitors, with a dietitian and a psychologist in attendance; a clinic stall with books and British Diabetic Association information packs; an active local parents group; arrangements for liaison with transfer of care to the counterpart adult clinic; and the routine practice of measuring blood pressure, screening for proteinuria, and examining the eyes.

Furthermore, clinical practice in the management of children with diabetes is changing in the wake of applied clinical research. New tests are beginning to define the early markers of diabetic complications in children, such as measures of heart rate variability, blood pressure response to 